\title{
Beth Levine receives the 2014 ASCI/Stanley J. Korsmeyer Award
}

A

utophagy is an evolutionarily conserved process that degrades large cellular components and plays a critical role in maintaining cellular homeostasis. This year, the ASCI recognizes seminal work in the field by Beth Levine (Figure 1) of the University of Texas Southwestern Medical Center with its annual Stanley J. Korsmeyer Award. The prize honors Levine for her groundbreaking studies that first elucidated the genetic control of autophagy in mammalian systems and subsequent work that has implicated the autophagy pathway in the pathogenesis of a wide spectrum of diseases, including cancer, aging, neurodegeneration, infectious diseases, and diabetes. Levine recently talked with the JCI to discuss her extraordinary path in research.

JCI: What first attracted you to a career in medicine and then in research?

Levine: I was originally attracted to a career in medicine for what I think is a typical reason: I wanted to help people. I found clinical medicine very gratifying from a personal and humanistic point of view. I loved clinical medicine, but I loved research even more. Research provided more opportunities for intellectual creativity and discovery and more opportunities to have a long-term impact on human disease. When you are caring for an individual patient, it is intellectually stimulating to try to understand what is going on and personally gratifying if you can use this understanding to help the patient. But you are only filling in the pieces of a small puzzle. There is no room for generating testable hypotheses to solve bigger puzzles in biology and medicine, which can potentially allow you to have a greater impact on more people.

JCI: Your early research focused on infectious diseases and viral pathogens. How did this line of study lead you to autophagy?

Levine: This actually ties to Stan Korsmeyer himself and his work on apoptosis. When I was a fellow, he was in the early days of his pioneering work on the role of BCL2 in the regulation of apoptosis and lymphoma. When I was a postdoctoral fellow, it occurred to me that we really did not know how viruses kill cells and why certain cells were resistant to lytic viral replication. So, using Sindbis virus, an alphavirus that provides an animal model for studying human arthropod-borne encephalitides, I showed that Sindbis virus killed cells by inducing apoptosis, that BCL2 blocked the process, and that BCL2 overexpression protected mice against lethal viral encephalitis. While

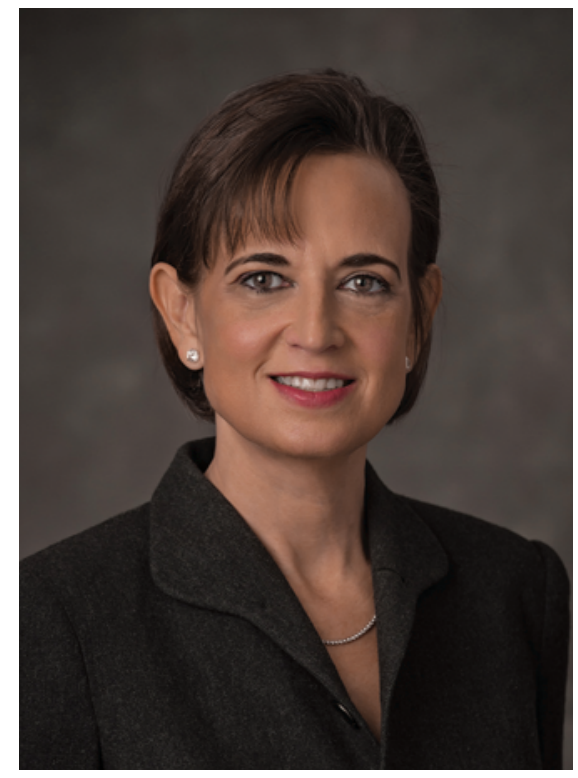

Figure 1

Beth Levine will be honored with the 2014 ASCI/Stanley J. Korsmeyer Award for her work on autophagy. Image credit: Brian Coats for UT Southwestern Medical Center.

investigating how BCL2 protected neurons against viral infection, we performed a yeast two-hybrid screen to identify novel BCL2-interacting proteins. This led to our identification of the protein that is now known as beclin 1 . Initially, all we knew was that beclin 1 interacted with BCL2 and was a predicted coiled-coil protein. The reason we became interested in studying beclin 1 and the reason I switched gears and broadened my research beyond infectious diseases - was that beclin 1 was allelically deleted (one copy of the gene was lost) in about $50 \%$ of patients with sporadic breast and ovarian cancers. This gave me the idea that this gene might be linked to cancer susceptibility. We had already shown that beclin 1 had antiviral effects in vivo in mice with Sindbis virus encephalitis. So our work on viruses and apoptosis led us to serendipitously discover beclin 1 , which we later demonstrated was a mammalian autophagy and tumor-suppressor protein. Once we demonstrated that beclin 1 functions in autophagy, antiviral host defense, and tumor suppression, I became really excited about further exploring possible roles of autophagy in different aspects of physiology and pathophysiology.

JCI: We now know that autophagy is a fundamental process that is deregulated in many different pathological settings. Did you appreciate early on the broad implications of the work?

Levine: Even before we knew beclin 1 was an autophagy gene, we thought that there might be implications for cancer. When we realized beclin 1 shared sequence similarities with a yeast autophagy gene, we examined whether beclin 1 was required for autophagy. We showed that beclin 1 functioned in mammalian autophagy and could restore autophagy in yeast lacking the orthologous gene. After this discovery, we thought about what autophagy might do. The process degrades unwanted things inside the cell that are too big to be degraded by the proteasome, and it is the only pathway to rid the cell of damaged organelles, microbes, and protein aggregates. Therefore, it made sense that autophagy might be involved in preventing aging and cancer by removing damaged organelles and preventing genotoxic stress; in innate immunity, by removing intracellular pathogens; and in preventing neurodegenerative diseases by removing mutant aggregate-prone proteins. It also made sense that it might be involved in development, a process that requires cellular architectural remodeling. Of course, in the early days, we did not know that autophagy was involved in these processes, but we had an intuition that it might be. And this is what motivated us to work in this area. When it became clear that the genes that mediated autophagy were evolutionarily conserved, we were able to use different model organisms to test our hypotheses. 
JCI: As your work extended across these divergent fields, what factors do you feel were most critical to your success?

Levine: I think what was most critical to my success was my willingness to follow my scientific intuition and curiosities and pursue questions that I thought were important - even though they were outside of my previously defined comfort zone or areas of expertise and even though it seemed to me that no one else thought they were important at the time. Also, I think my excitement about integrating concepts from different disciplines to generate new hypotheses about interrelationships between a cell's biological pathway and diseases was important to my success.

JCI: Looking back, were there obstacles to getting where you are now?

Levine: First, I should say that I have been very fortunate to be able to develop my scientific career at Columbia and at UT Southwestern. These two institutions have outstanding intellectual environments, and at both institutions, the leadership encouraged and valued my work. I was given complete scientific freedom to explore my ideas, even though they did not fit within the conventional framework of what an infectious diseases-trained physician-scientist should do. Without this freedom and support, I could not have gotten to where I am today.

I think the major obstacle - at the time we first discovered beclin 1 and linked it to autophagy - was simply that most scientists had not yet heard of autophagy. Even when you explained the process, most people did not think it was important, perhaps because there had not been a long history of previous work on mammalian autophagy. Although there were studies showing that the yeast genes were essential for survival during starvation, almost nothing was known about the functions of autophagy in higher eukaryotic organisms.
That made it difficult initially to get our grants funded and our papers published.

Our 1999 paper in Nature describing a role of beclin 1 in autophagy and tumor suppression was probably the hardest paper I have ever had to get accepted (1). At that time, the scientific community did not widely recognize the concept of a haploinsufficient tumor suppressor. We had data that beclin 1 had tumor-suppressor function in breast cancer cells long before we published our 1999 Nature paper, but the gold standard at that time was that both alleles needed to be inactivated to show that a gene was a tumor suppressor. We studied breast and ovarian cancers from patients who had monoallelic loss of beclin 1 and feverishly sequenced the remaining allele, but did not find any inactivating mutations. As beclin 1 did not fulfill the classical "Knudson two-hit hypothesis" definition of a tumor suppressor, it was challenging in the early days to prove the cancer connection to other scientists.

JCI: Your 2013 Nature paper reported the identification of an autophagy-inducing peptide (2). Are you hopeful about the prospect of translating autophagy research into the clinic?

Levine: Yes, I am cautiously very optimistic that we and other researchers will be able to develop pharmacological ways to upregulate autophagy in order to treat a variety of human diseases. However, as is often the case when you try to target a broad pathway that has multiple functions in the cell and organism, it may not be straightforward. Our 2012 Nature paper on exercise suggests that exercise may have beneficial health effects partly through upregulation of autophagy (3). Earlier work from our lab showed that autophagy mediates the longevity effects of caloric restriction in nematodes. Thus, exercise and caloric restriction may be two physiological stimuli that upregulate autophagy to promote health and longevity. But such lifestyle changes are difficult to make and may be insufficient for clinical purposes. Therefore, there is a real need to develop specific therapies that can increase autophagy to prevent or treat certain infectious diseases, neurodegenerative disorders, cancer, metabolic diseases, and inflammatory conditions. So we are working on developing our new autophagy-inducing peptide into a drug-like molecule and expect to see it eventually tested in patients. Many other labs are working on additional approaches to modulate autophagy to treat different diseases.

JCI: The ASCI's honor of the Korsmeyer Award follows on the heels of your election to the National Academy of Sciences in 2013 and the AAAS in 2012. What have these accolades meant to you?

Levine: These accolades recognize the importance of autophagy as a crucial pathway in health and disease and thus really reflect the accomplishments of many researchers in the field of autophagy. On a personal level, it is rewarding to know that perseverance based on intuition, the joy of scientific curiosity and discovery, and the hard work of many great people in my lab and those of my colleagues is now being recognized by the biomedical community. As an example to young people, it is really gratifying to know that if you work hard, follow your scientific instincts, and struggle successfully through the challenging moments, you can potentially achieve important things.

\section{Sarah Jackson}

1. Liang $\mathrm{XH}$, et al. Induction of autophagy and inhibition of tumorigenesis by beclin 1. Nature. 1999;402(6762):672-676.

2. Shoji-Kawata $S$, et al. Identification of a candidate therapeutic autophagy-inducing peptide. Nature. 2013;494(7436):201-206.

3. He C, et al. Exercise-induced BCL2-regulated autophagy is required for muscle glucose homeostasis. Nature. 2012;481(7382):511-515. 